\title{
Inversion Formulas for the Dunkl Intertwining Operator and Its Dual on Spaces of Functions and Distributions*
}

\author{
Khalifa TRIMÈCHE
}

Faculty of Sciences of Tunis, Department of Mathematics, 1060 Tunis, Tunisia

E-mail: Khlifa.trimeche@fst.rnu.tn

Received May 13, 2008, in final form September 16, 2008; Published online September 29, 2008

Original article is available at http://www.emis.de/journals/SIGMA/2008/067/

\begin{abstract}
In this paper we prove inversion formulas for the Dunkl intertwining operator $V_{k}$ and for its dual ${ }^{t} V_{k}$ and we deduce the expression of the representing distributions of the inverse operators $V_{k}^{-1}$ and ${ }^{t} V_{k}^{-1}$, and we give some applications.
\end{abstract}

Key words: inversion formulas; Dunkl intertwining operator; dual Dunkl intertwining operator

2000 Mathematics Subject Classification: 33C80; 43A32; 44A35; 51F15

\section{Introduction}

We consider the differential-difference operators $T_{j}, j=1,2, \ldots, d$, on $\mathbb{R}^{d}$ associated to a root system $R$ and a multiplicity function $k$, introduced by C.F. Dunkl in [3] and called the Dunkl operators in the literature. These operators are very important in pure mathematics and in physics. They provide a useful tool in the study of special functions related to root systems $[4,6,2]$. Moreover the commutative algebra generated by these operators has been used in the study of certain exactly solvable models of quantum mechanics, namely the Calogero-Sutherland-Moser models, which deal with systems of identical particles in a one dimensional space (see $[8,11,12])$.

C.F. Dunkl proved in [4] that there exists a unique isomorphism $V_{k}$ from the space of homogeneous polynomials $\mathcal{P}_{n}$ on $\mathbb{R}^{d}$ of degree $n$ onto itself satisfying the transmutation relations

$$
T_{j} V_{k}=V_{k} \frac{\partial}{\partial x_{j}}, \quad j=1,2, \ldots, d,
$$

and

$$
V_{k}(1)=1
$$

This operator is called the Dunkl intertwining operator. It has been extended to an isomorphism from $\mathcal{E}\left(\mathbb{R}^{d}\right)$ (the space of $C^{\infty}$-functions on $\mathbb{R}^{d}$ ) onto itself satisfying the relations (1.1) and (1.2) (see $[15])$.

The operator $V_{k}$ possesses the integral representation

$$
\forall x \in \mathbb{R}^{d}, \quad V_{k}(f)(x)=\int_{\mathbb{R}^{d}} f(y) d \mu_{x}(y), \quad f \in \mathcal{E}\left(\mathbb{R}^{d}\right),
$$

where $\mu_{x}$ is a probability measure on $\mathbb{R}^{d}$ with support in the closed ball $B(0,\|x\|)$ of center 0 and radius $\|x\|$ (see $[14,15])$.

\footnotetext{
${ }^{\star}$ This paper is a contribution to the Special Issue on Dunkl Operators and Related Topics. The full collection is available at http://www.emis.de/journals/SIGMA/Dunkl_operators.html
} 
We have shown in [15] that for each $x \in \mathbb{R}^{d}$, there exists a unique distribution $\eta_{x}$ in $\mathcal{E}^{\prime}\left(\mathbb{R}^{d}\right)$ (the space of distributions on $\mathbb{R}^{d}$ of compact support) with support in $B(0,\|x\|)$ such that

$$
V_{k}^{-1}(f)(x)=\left\langle\eta_{x}, f\right\rangle, \quad f \in \mathcal{E}\left(\mathbb{R}^{d}\right) .
$$

We have studied also in [15] the transposed operator ${ }^{t} V_{k}$ of the operator $V_{k}$, satisfying for $f$ in $\mathcal{S}\left(\mathbb{R}^{d}\right.$ ) (the space of $C^{\infty}$-functions on $\mathbb{R}^{d}$ which are rapidly decreasing together with their derivatives) and $g$ in $\mathcal{E}\left(\mathbb{R}^{d}\right)$, the relation

$$
\int_{\mathbb{R}^{d}}{ }^{t} V_{k}(f)(y) g(y) d y=\int_{\mathbb{R}^{d}} V_{k}(g)(x) f(x) \omega_{k}(x) d x,
$$

where $\omega_{k}$ is a positive weight function on $\mathbb{R}^{d}$ which will be defined in the following section. It has the integral representation

$$
\forall y \in \mathbb{R}^{d}, \quad{ }^{t} V_{k}(f)(y)=\int_{\mathbb{R}^{d}} f(x) d \nu_{y}(x),
$$

where $\nu_{y}$ is a positive measure on $\mathbb{R}^{d}$ with support in the set $\left\{x \in \mathbb{R}^{d} ;\|x\| \geq\|y\|\right\}$. This operator is called the dual Dunkl intertwining operator.

We have proved in [15] that the operator ${ }^{t} V_{k}$ is an isomorphism from $\mathcal{D}\left(\mathbb{R}^{d}\right)$ (the space of $C^{\infty}$ functions on $\mathbb{R}^{d}$ with compact support) (resp. $\mathcal{S}\left(\mathbb{R}^{d}\right)$ ) onto itself, satisfying the transmutation relations

$$
\forall y \in \mathbb{R}^{d}, \quad{ }^{t} V_{k}\left(T_{j} f\right)(y)=\frac{\partial}{\partial y_{j}}{ }^{t} V_{k}(f)(y), \quad j=1,2, \ldots, d .
$$

Moreover for each $y \in \mathbb{R}^{d}$, there exists a unique distribution $Z_{y}$ in $\mathcal{S}^{\prime}\left(\mathbb{R}^{d}\right)$ (the space of tempered distributions on $\mathbb{R}^{d}$ ) with support in the set $\left\{x \in \mathbb{R}^{d} ;\|x\| \geq\|y\|\right\}$ such that

$$
{ }^{t} V_{k}^{-1}(f)(y)=\left\langle Z_{y}, f\right\rangle, \quad f \in \mathcal{S}\left(\mathbb{R}^{d}\right) .
$$

Using the operator $V_{k}$, C.F. Dunkl has defined in [5] the Dunkl kernel $K$ by

$$
\forall x \in \mathbb{R}^{d}, \quad \forall z \in \mathbb{C}^{d}, \quad K(x,-i z)=V_{k}\left(e^{-i\langle\cdot, z\rangle}\right)(x) .
$$

Using this kernel C.F. Dunkl has introduced in [5] a Fourier transform $\mathcal{F}_{D}$ called the Dunkl transform.

In this paper we establish the following inversion formulas for the operators $V_{k}$ and ${ }^{t} V_{k}$ :

$$
\begin{array}{ll}
\forall x \in \mathbb{R}^{d}, & V_{k}^{-1}(f)(x)=P^{t} V_{k}(f)(x), \quad f \in \mathcal{S}\left(\mathbb{R}^{d}\right), \\
\forall x \in \mathbb{R}^{d}, & { }^{t} V_{k}^{-1}(f)(x)=V_{k}(P(f))(x), \quad f \in \mathcal{S}\left(\mathbb{R}^{d}\right),
\end{array}
$$

where $P$ is a pseudo-differential operator on $\mathbb{R}^{d}$.

When the multiplicity function takes integer values, the formula (1.8) can also be written in the form

$$
\forall x \in \mathbb{R}^{d}, \quad V_{k}^{-1}(f)(x)={ }^{t} V_{k}(Q(f))(x), \quad f \in \mathcal{S}\left(\mathbb{R}^{d}\right),
$$

where $Q$ is a differential-difference operator.

Also we give another expression of the operator ${ }^{t} V_{k}^{-1}$ on the space $\mathcal{E}^{\prime}\left(\mathbb{R}^{d}\right)$. From these relations we deduce the expressions of the representing distributions $\eta_{x}$ and $Z_{x}$ of the inverse operators $V_{k}^{-1}$ and ${ }^{t} V_{k}^{-1}$ by using the representing measures $\mu_{x}$ and $\nu_{x}$ of $V_{k}$ and ${ }^{t} V_{k}$. They are given by the following formulas

$$
\begin{array}{ll}
\forall x \in \mathbb{R}^{d}, & \eta_{x}={ }^{t} Q\left(\nu_{x}\right), \\
\forall x \in \mathbb{R}^{d}, & Z_{x}={ }^{t} P\left(\mu_{x}\right),
\end{array}
$$

where ${ }^{t} P$ and ${ }^{t} Q$ are the transposed operators of $P$ and $Q$ respectively. 
The contents of the paper are as follows. In Section 2 we recall some basic facts from Dunkl's theory, and describe the Dunkl operators and the Dunkl kernel. We define in Section 3 the Dunkl transform introduced in [5] by C.F. Dunkl, and we give the main theorems proved for this transform, which will be used in this paper. We study in Section 4 the Dunkl convolution product and the Dunkl transform of distributions which will be useful in the sequel, and when the multiplicity function takes integer values, we give another proof of the geometrical form of Paley-Wiener-Schwartz theorem for the Dunkl transform. We prove in Section 5 some inversion formulas for the Dunkl intertwining operator $V_{k}$ and its dual ${ }^{t} V_{k}$ on spaces of functions and distributions. Section 6 is devoted to proving under the condition that the multiplicity function takes integer values an inversion formula for the Dunkl intertwining operator $V_{k}$, and we deduce the expression of the representing distributions of the inverse operators $V_{k}^{-1}$ and ${ }^{t} V_{k}^{-1}$. In Section 7 we give some applications of the preceding inversion formulas.

\section{The eigenfunction of the Dunkl operators}

In this section we collect some notations and results on the Dunkl operators and the Dunkl kernel (see $[3,4,5,7,9,10])$.

\subsection{Reflection groups, root systems and multiplicity functions}

We consider $\mathbb{R}^{d}$ with the Euclidean scalar product $\langle\cdot, \cdot\rangle$ and $\|x\|=\sqrt{\langle x, x\rangle}$. On $\mathbb{C}^{d},\|\cdot\|$ denotes also the standard Hermitian norm, while $\langle z, w\rangle=\sum_{j=1}^{d} z_{j} \overline{w_{j}}$.

For $\alpha \in \mathbb{R}^{d} \backslash\{0\}$, let $\sigma_{\alpha}$ be the reflection in the hyperplane $H_{\alpha} \subset \mathbb{R}^{d}$ orthogonal to $\alpha$, i.e.

$$
\sigma_{\alpha}(x)=x-\left(\frac{2\langle\alpha, x\rangle}{\|\alpha\|^{2}}\right) \alpha .
$$

A finite set $R \subset \mathbb{R}^{d} \backslash\{0\}$ is called a root system if $R \cap \mathbb{R} \alpha=\{ \pm \alpha\}$ and $\sigma_{\alpha} R=R$ for all $\alpha \in R$. For a given root system $R$ the reflections $\sigma_{\alpha}, \alpha \in R$, generate a finite group $W \subset O(d)$, the reflection group associated with $R$. All reflections in $W$ correspond to suitable pairs of roots. For a given $\beta \in \mathbb{R}^{d} \backslash \cup_{\alpha \in R} H_{\alpha}$, we fix the positive subsystem $R_{+}=\{\alpha \in R ;\langle\alpha, \beta\rangle>0\}$, then for each $\alpha \in R$ either $\alpha \in R_{+}$or $-\alpha \in R_{+}$.

A function $k: R \rightarrow \mathbb{C}$ on a root system $R$ is called a multiplicity function if it is invariant under the action of the associated reflection group $W$. If one regards $k$ as a function on the corresponding reflections, this means that $k$ is constant on the conjugacy classes of reflections in $W$. For abbreviation, we introduce the index

$$
\gamma=\gamma(R)=\sum_{\alpha \in R_{+}} k(\alpha)
$$

Moreover, let $\omega_{k}$ denotes the weight function

$$
\omega_{k}(x)=\prod_{\alpha \in R_{+}}|\langle\alpha, x\rangle|^{2 k(\alpha)},
$$

which is $W$-invariant and homogeneous of degree $2 \gamma$.

For $d=1$ and $W=\mathbb{Z}_{2}$, the multiplicity function $k$ is a single parameter denoted $\gamma$ and

$$
\forall x \in \mathbb{R}, \quad \omega_{k}(x)=|x|^{2 \gamma} .
$$

We introduce the Mehta-type constant

$$
c_{k}=\left(\int_{\mathbb{R}^{d}} e^{-\|x\|^{2}} \omega_{k}(x) d x\right)^{-1},
$$

which is known for all Coxeter groups $W$ (see $[3,6])$. 


\subsection{The Dunkl operators and the Dunkl kernel}

The Dunkl operators $T_{j}, j=1, \ldots, d$, on $\mathbb{R}^{d}$, associated with the finite reflection group $W$ and the multiplicity function $k$, are given for a function $f$ of class $C^{1}$ on $\mathbb{R}^{d}$ by

$$
T_{j} f(x)=\frac{\partial}{\partial x_{j}} f(x)+\sum_{\alpha \in R_{+}} k(\alpha) \alpha_{j} \frac{f(x)-f\left(\sigma_{\alpha}(x)\right)}{\langle\alpha, x\rangle} .
$$

In the case $k \equiv 0$, the $T_{j}, j=1,2, \ldots, d$, reduce to the corresponding partial derivatives. In this paper, we will assume throughout that $k \geq 0$ and $\gamma>0$.

For $f$ of class $C^{1}$ on $\mathbb{R}^{d}$ with compact support and $g$ of class $C^{1}$ on $\mathbb{R}^{d}$ we have

$$
\int_{\mathbb{R}^{d}} T_{j} f(x) g(x) \omega_{k}(x) d x=-\int_{\mathbb{R}^{d}} f(x) T_{j} g(x) \omega_{k}(x) d x, \quad j=1,2, \ldots, d .
$$

For $y \in \mathbb{R}^{d}$, the system

$$
\begin{aligned}
& T_{j} u(x, y)=y_{j} u(x, y), \quad j=1,2, \ldots, d, \\
& u(0, y)=1,
\end{aligned}
$$

admits a unique analytic solution on $\mathbb{R}^{d}$, denoted by $K(x, y)$ and called the Dunkl kernel.

This kernel has a unique holomorphic extension to $\mathbb{C}^{d} \times \mathbb{C}^{d}$.

Example 2.1. From [5], if $d=1$ and $W=\mathbb{Z}_{2}$, the Dunkl kernel is given by

$$
K(z, t)=j_{\gamma-1 / 2}(i z t)+\frac{z t}{2 \gamma+1} j_{\gamma+1 / 2}(i z t), \quad z, t \in \mathbb{C},
$$

where for $\alpha \geq-1 / 2, j_{\alpha}$ is the normalized Bessel function defined by

$$
j_{\alpha}(u)=2^{\alpha} \Gamma(\alpha+1) \frac{J_{\alpha}(u)}{u^{\alpha}}=\Gamma(\alpha+1) \sum_{n=0}^{\infty} \frac{(-1)^{n}(u / 2)^{2 n}}{n ! \Gamma(n+\alpha+1)}, \quad u \in \mathbb{C},
$$

with $J_{\alpha}$ being the Bessel function of first kind and index $\alpha$ (see [16]).

The Dunkl kernel possesses the following properties.

(i) For $z, t \in \mathbb{C}^{d}$, we have $K(z, t)=K(t, z), K(z, 0)=1$, and $K(\lambda z, t)=K(z, \lambda t)$ for all $\lambda \in \mathbb{C}$.

(ii) For all $\nu \in \mathbb{Z}_{+}^{d}, x \in \mathbb{R}^{d}$, and $z \in \mathbb{C}^{d}$ we have

$$
\left|D_{z}^{\nu} K(x, z)\right| \leq\|x\|^{|\nu|} \exp \left[\max _{w \in W}\langle w x, \operatorname{Re} z\rangle\right] .
$$

with

$$
D_{z}^{\nu}=\frac{\partial^{|\nu|}}{\partial z_{1}^{\nu_{1}} \cdots \partial z_{d}^{\nu_{d}}} \quad \text { and } \quad|\nu|=\nu_{1}+\cdots+\nu_{d}
$$

In particular

$$
\begin{aligned}
& \left.\left|D_{z}^{\nu} K(x, z)\right| \leq\|x\|^{|\nu|} \exp [\|x\|\|\operatorname{Re} z\|]\right] \\
& |K(x, z)| \leq \exp [\|x\|\|\operatorname{Re} z\|]
\end{aligned}
$$

and for all $x, y \in \mathbb{R}^{d}$

$$
|K(i x, y)| \leq 1
$$


(iii) For all $x, y \in \mathbb{R}^{d}$ and $w \in W$ we have

$$
K(-i x, y)=\overline{K(i x, y)} \quad \text { and } \quad K(w x, w y)=K(x, y) .
$$

(iv) The function $K(x, z)$ admits for all $x \in \mathbb{R}^{d}$ and $z \in \mathbb{C}^{d}$ the following Laplace type integral representation

$$
K(x, z)=\int_{\mathbb{R}^{d}} e^{\langle y, z\rangle} d \mu_{x}(y),
$$

where $\mu_{x}$ is the measure given by the relation (1.3) (see [14]).

Remark 2.1. When $d=1$ and $W=\mathbb{Z}_{2}$, the relation (2.6) is of the form

$$
K(x, z)=\frac{\Gamma(\gamma+1 / 2)}{\sqrt{\pi} \Gamma(\gamma)}|x|^{-2 \gamma} \int_{-|x|}^{|x|}(|x|-y)^{\gamma-1}(|x|+y)^{\gamma} e^{y z} d y .
$$

Then in this case the measure $\mu_{x}$ is given for all $x \in \mathbb{R} \backslash\{0\}$ by $d \mu_{x}(y)=\mathcal{K}(x, y) d y$ with

$$
\mathcal{K}(x, y)=\frac{\Gamma(\gamma+1 / 2)}{\sqrt{\pi} \Gamma(\gamma)}|x|^{-2 \gamma}(|x|-y)^{\gamma-1}(|x|+y)^{\gamma} 1_{]-|x|,|x|[}(y),
$$

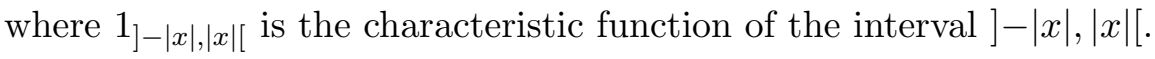

\section{The Dunkl transform}

In this section we define the Dunkl transform and we give the main results satisfied by this transform which will be used in the following sections (see $[5,9,10]$ ).

Notation. We denote by $\mathbb{H}\left(\mathbb{C}^{d}\right)$ the space of entire functions on $\mathbb{C}^{d}$ which are rapidly decreasing and of exponential type. We equip this space with the classical topology.

The Dunkl transform of a function $f$ in $\mathcal{S}\left(\mathbb{R}^{d}\right)$ is given by

$$
\forall y \in \mathbb{R}^{d}, \quad \mathcal{F}_{D}(f)(y)=\int_{\mathbb{R}^{d}} f(x) K(x,-i y) \omega_{k}(x) d x .
$$

This transform satisfies the relation

$$
\mathcal{F}_{D}(f)=\mathcal{F} \circ{ }^{t} V_{k}(f), \quad f \in \mathcal{S}\left(\mathbb{R}^{d}\right),
$$

where $\mathcal{F}$ is the classical Fourier transform on $\mathbb{R}^{d}$ given by

$$
\forall y \in \mathbb{R}^{d}, \quad \mathcal{F}(f)(y)=\int_{\mathbb{R}^{d}} f(x) e^{-i\langle x, y\rangle} d x, \quad f \in \mathcal{S}\left(\mathbb{R}^{d}\right) .
$$

The following theorems are proved in $[9,10]$.

Theorem 3.1. The transform $\mathcal{F}_{D}$ is a topological isomorphism

i) from $\mathcal{D}\left(\mathbb{R}^{d}\right)$ onto $\mathbb{H}\left(\mathbb{C}^{d}\right)$,

ii) from $\mathcal{S}\left(\mathbb{R}^{d}\right)$ onto itself.

The inverse transform is given by

$$
\forall x \in \mathbb{R}^{d}, \quad \mathcal{F}_{D}^{-1}(h)(x)=\frac{c_{k}^{2}}{2^{2 \gamma+d}} \int_{\mathbb{R}^{d}} h(y) K(x, i y) \omega_{k}(y) d y .
$$


Remark 3.1. Another proof of Theorem 3.1 is given in [17].

When the multiplicity function satisfies $k(\alpha) \in \mathbb{N}$ for all $\alpha \in R_{+}$, M.F.E. de Jeu has proved in [10] the following geometrical form of Paley-Wiener theorem for functions.

Theorem 3.2. Let $E$ be a $W$-invariant compact convex set of $\mathbb{R}^{d}$ and $f$ an entire function on $\mathbb{C}^{d}$. Then $f$ is the Dunkl transform of a function in $\mathcal{D}\left(\mathbb{R}^{d}\right)$ with support in $E$, if and only if for all $q \in \mathbb{N}$ there exists a positive constant $C_{q}$ such that

$$
\forall z \in \mathbb{C}^{d}, \quad|f(z)| \leq C_{q}(1+\|z\|)^{-q} e^{I_{E}(\operatorname{Im} z)},
$$

where $I_{E}$ is the gauge associated to the polar of $E$, given by

$$
\forall y \in \mathbb{R}^{d}, \quad I_{E}(y)=\sup _{x \in E}\langle x, y\rangle .
$$

\section{The Dunkl convolution product and the Dunkl transform of distributions}

\subsection{The Dunkl translation operators and the Dunkl convolution product of functions}

The definitions and properties of the Dunkl translation operators and the Dunkl convolution product of functions presented in this subsection are given in the seventh section of [17, pages $33-37]$.

The Dunkl translation operators $\tau_{x}, x \in \mathbb{R}^{d}$, are defined on $\mathcal{E}\left(\mathbb{R}^{d}\right)$ by

$$
\forall y \in \mathbb{R}^{d}, \quad \tau_{x} f(y)=\left(V_{k}\right)_{x}\left(V_{k}\right)_{y}\left[V_{k}^{-1}(f)(x+y)\right] .
$$

For $f$ in $\mathcal{S}\left(\mathbb{R}^{d}\right)$ the function $\tau_{x} f$ can also be written in the form

$$
\forall y \in \mathbb{R}^{d}, \quad \tau_{x} f(y)=\left(V_{k}\right)_{x}\left({ }^{t} V_{k}^{-1}\right)_{y}\left[{ }^{t} V_{k}(f)(x+y)\right] .
$$

Using properties of the operators $V_{k}$ and ${ }^{t} V_{k}$ we deduce that for $f$ in $\mathcal{D}\left(\mathbb{R}^{d}\right)$ (resp. $\mathcal{S}\left(\mathbb{R}^{d}\right)$ ) and $x \in \mathbb{R}^{d}$, the function $y \rightarrow \tau_{x} f(y)$ belongs to $\mathcal{D}\left(\mathbb{R}^{d}\right)\left(\right.$ resp. $\left.\mathcal{S}\left(\mathbb{R}^{d}\right)\right)$ and we have

$$
\forall t \in \mathbb{R}^{d}, \quad \mathcal{F}_{D}\left(\tau_{x} f\right)(t)=K(i x, t) \mathcal{F}_{D}(f)(t) .
$$

The Dunkl convolution product of $f$ and $g$ in $\mathcal{D}\left(\mathbb{R}^{d}\right)$ is the function $f *_{D} g$ defined by

$$
\forall x \in \mathbb{R}^{d}, \quad f *_{D} g(x)=\int_{\mathbb{R}^{d}} \tau_{x} f(-y) g(y) \omega_{k}(y) d y .
$$

For $f, g$ in $\mathcal{D}\left(\mathbb{R}^{d}\right)\left(\right.$ resp. $\left.\mathcal{S}\left(\mathbb{R}^{d}\right)\right)$ the function $f *_{D} g$ belongs to $\mathcal{D}\left(\mathbb{R}^{d}\right)\left(\right.$ resp. $\left.\mathcal{S}\left(\mathbb{R}^{d}\right)\right)$ and we have

$$
\forall t \in \mathbb{R}^{d}, \quad \mathcal{F}_{D}\left(f *_{D} g\right)(t)=\mathcal{F}_{D}(f)(t) \mathcal{F}_{D}(g)(t) .
$$

\subsection{The Dunkl convolution product of tempered distributions}

Definition 4.1. Let $S$ be in $\mathcal{S}^{\prime}\left(\mathbb{R}^{d}\right)$ and $\varphi$ in $\mathcal{S}\left(\mathbb{R}^{d}\right)$. The Dunkl convolution product of $S$ and $\varphi$ is the function $S *_{D} \varphi$ defined by

$$
\forall x \in \mathbb{R}^{d}, \quad S *_{D} \varphi(x)=\left\langle S_{y}, \tau_{x} \varphi(-y)\right\rangle .
$$


Proposition 4.1. For $S$ in $\mathcal{S}^{\prime}\left(\mathbb{R}^{d}\right)$ and $\varphi$ in $\mathcal{S}\left(\mathbb{R}^{d}\right)$ the function $S *_{D} \varphi$ belongs to $\mathcal{E}\left(\mathbb{R}^{d}\right)$ and we have

$$
T^{\mu}\left(S *_{D} \varphi\right)=S *_{D}\left(T^{\mu}(\varphi)\right)
$$

where

$$
T^{\mu}=T_{1}^{\mu_{1}} \circ T_{2}^{\mu_{2}} \circ \cdots \circ T_{d}^{\mu_{d}} \quad \text { with } \quad \mu=\left(\mu_{1}, \mu_{2}, \ldots, \mu_{d}\right) \in \mathbb{N}^{d} .
$$

Proof. We remark first that the topology of $\mathcal{S}\left(\mathbb{R}^{d}\right)$ is also generated by the seminorms

$$
Q_{k, l}(\psi)=\sup _{\substack{|\mu| \leq k \\ x \in \mathbb{R}^{d}}}\left(1+\|x\|^{2}\right)^{l}\left|T^{\mu} \psi(x)\right|, \quad k, l \in \mathbb{N} .
$$

i) Let $x_{0} \in \mathbb{R}^{d}$. We prove first that $S *_{D} \varphi$ is continuous at $x_{0}$. We have

$$
\forall x \in \mathbb{R}^{d}, \quad S *_{D} \varphi(x)-S *_{D} \varphi\left(x_{0}\right)=\left\langle S_{y},\left(\tau_{x} \varphi-\tau_{x_{0}} \varphi\right)(-y)\right\rangle .
$$

We must prove that $\left(\tau_{x} \varphi-\tau_{x_{0}} \varphi\right)$ converges to zero in $\mathcal{S}\left(\mathbb{R}^{d}\right)$ when $x$ tends to $x_{0}$.

Let $k, \ell \in \mathbb{N}$ and $\mu \in \mathbb{N}^{d}$ such that $|\mu| \leq k$. From (4.3), Theorem 3.1 and the relations (2.1), (2.2) we have

$$
\begin{aligned}
(1+ & \left.\|y\|^{2}\right)^{\ell} T^{\mu}\left(\tau_{x} \varphi-\tau_{x_{0}} \varphi\right)(-y) \\
& =\frac{i^{|\mu|} c_{k}^{2}}{2^{2 \gamma+d}} \int_{\mathbb{R}^{d}}\left(1+\|\lambda\|^{2}\right)^{p} K(i \lambda,-y)\left(I-\Delta_{k}\right)^{\ell}\left[\lambda^{\mu}(K(-i x, \lambda)\right. \\
& \left.\left.-K\left(-i x_{0}, \lambda\right)\right) \mathcal{F}_{D}(\varphi)(\lambda)\right] \frac{\omega_{k}(\lambda)}{\left(1+\|\lambda\|^{2}\right)^{p}} d \lambda,
\end{aligned}
$$

with $\lambda^{\mu}=\lambda_{1}^{\mu_{1}} \lambda_{2}^{\mu_{2}} \cdots \lambda_{d}^{\mu_{d}}, \Delta_{k}=\sum_{j=1}^{d} T_{j}^{2}$ the Dunkl Laplacian and $p \in \mathbb{N}$ such that $p>\gamma+\frac{d}{2}+1$.

Using (2.4) and (2.5) we deduce that

$$
Q_{k, \ell}\left(\tau_{x} \varphi-\tau_{x_{0}} \varphi\right)=\sup _{\substack{|\mu| \leq k \\ y \in \mathbb{R}^{d}}}(1+\|y\|)^{\ell}\left|T^{\mu}\left(\tau_{x} \varphi-\tau_{x_{0}} \varphi\right)(-y)\right| \rightarrow 0 \quad \text { as } \quad x \rightarrow x_{0} .
$$

Then the function $S *_{D} \varphi$ is continuous at $x_{0}$, and thus it is continuous on $\mathbb{R}^{d}$.

Now we will prove that $S *_{D} \varphi$ admits a partial derivative on $\mathbb{R}^{d}$ with respect to the variable $x_{j}$. Let $h \in \mathbb{R} \backslash\{0\}$. We consider the function $f_{h}$ defined on $\mathbb{R}^{d}$ by

$$
f_{h}(y)=\frac{1}{h}\left(\tau_{\left(x_{1}, \ldots, x_{j}+h, \ldots, x_{d}\right)} \varphi(-y)-\tau_{\left(x_{1}, \ldots, x_{j}, \ldots, x_{d}\right)} \varphi(-y)\right)-\frac{\partial}{\partial x_{j}} \tau_{x} \varphi(-y) .
$$

Using the formula

$$
\forall y \in \mathbb{R}^{d}, f_{h}(y)=\frac{1}{h} \int_{x_{j}}^{x_{j}+h}\left(\int_{x_{j}}^{u_{j}} \frac{\partial^{2}}{\partial t_{j}^{2}} \tau_{\left(x_{1}, \ldots, t_{j}, \ldots, x_{d}\right)} \varphi(-y) d t_{j}\right) d u_{j},
$$

we obtain for all $k, \ell \in \mathbb{N}$ and $\mu \in \mathbb{N}^{d}$ such that $|\mu| \leq k$ :

$$
\begin{aligned}
\forall y \in \mathbb{R}^{d}, \quad & \left(1+\|y\|^{2}\right)^{\ell} T^{\mu} f_{h}(y) \\
& =\frac{1}{h} \int_{x_{j}}^{x_{j}+h}\left(\int_{x_{j}}^{u_{j}}\left(1+\|y\|^{2}\right)^{\ell} T^{\mu} \frac{\partial^{2}}{\partial t_{j}^{2}} \tau_{\left(x_{1}, \ldots, t_{j}, x_{d}\right)} \varphi(-y) d t_{j}\right) d u_{j} .
\end{aligned}
$$


By applying the preceding method to the function

$$
\left(1+\|y\|^{2}\right)^{\ell} T^{\mu} \frac{\partial^{2}}{\partial t_{j}^{2}} \tau_{\left(x_{1}, \ldots, t_{j}, \ldots, x_{d}\right)} \varphi(-y),
$$

we deduce from the relation (4.4) that

$$
Q_{k, \ell}\left(f_{h}\right)=\sup _{\substack{|\mu| \leq k \\ y \in \mathbb{R}^{d}}}\left(1+\|y\|^{2}\right)^{\ell}\left|T^{\mu} f_{h}(y)\right| \rightarrow 0 \quad \text { as } \quad h \rightarrow 0 .
$$

Thus the function $S *_{D} \varphi(x)$ admits a partial derivative at $x_{0}$ with respect to $x_{j}$ and we have

$$
\frac{\partial}{\partial x_{j}} S *_{D} \varphi\left(x_{0}\right)=\left\langle S_{y}, \frac{\partial}{\partial x_{j}} \tau_{x_{0}} \varphi(-y)\right\rangle
$$

These results is true on $\mathbb{R}^{d}$. Moreover the partial derivatives are continuous on $\mathbb{R}^{d}$. By proceeding in a similar way for partial derivatives of all order with respect to all variables, we deduce that $S *_{D} \varphi$ belongs to $\mathcal{E}\left(\mathbb{R}^{d}\right)$.

ii) From the i) we have

$$
\forall x \in \mathbb{R}^{d}, \quad \frac{\partial}{\partial x_{j}} S *_{D} \varphi(x)=\left\langle S_{y}, \frac{\partial}{\partial x_{j}} \tau_{x} \varphi(-y)\right\rangle .
$$

On the other hand using the definition of the Dunkl operator $T_{j}$ and the relation

$$
T_{j}\left(\tau_{x} \varphi(-y)\right)=\tau_{x}\left(T_{j} \varphi\right)(-y),
$$

we obtain

$$
\forall x \in \mathbb{R}^{d}, \quad T_{j}\left(S *_{D} \varphi\right)(x)=\left\langle S_{y}, \tau_{x}\left(T_{j} \varphi\right)(-y)\right\rangle=S *_{D}\left(T_{j} \varphi\right)(x) .
$$

By iteration we get

$$
\forall x \in \mathbb{R}^{d}, \quad T^{\mu}\left(S *_{D} \varphi\right)(x)=S *_{D}\left(T^{\mu} \varphi\right)(x) .
$$

\subsection{The Dunkl transform of distributions}

\section{Definition 4.2.}

i) The Dunkl transform of a distribution $S$ in $\mathcal{S}^{\prime}\left(\mathbb{R}^{d}\right)$ is defined by

$$
\left\langle\mathcal{F}_{D}(S), \psi\right\rangle=\left\langle S, \mathcal{F}_{D}(\psi)\right\rangle, \psi \in \mathcal{S}\left(\mathbb{R}^{d}\right)
$$

ii) We define the Dunkl transform of a distribution $S$ in $\mathcal{E}^{\prime}\left(\mathbb{R}^{d}\right)$ by

$$
\forall y \in \mathbb{R}^{d}, \quad \mathcal{F}_{D}(S)(y)=\left\langle S_{x}, K(-i y, x)\right\rangle .
$$

Remark 4.1. When the distribution $S$ in $\mathcal{E}^{\prime}\left(\mathbb{R}^{d}\right)$ is given by the function $g \omega_{k}$ with $g$ in $\mathcal{D}\left(\mathbb{R}^{d}\right)$, and denoted by $T_{g \omega_{k}}$, the relation (4.5) coincides with (3.1).

Notation. We denote by $\mathcal{H}\left(\mathbb{C}^{d}\right)$ the space of entire functions on $\mathbb{C}^{d}$ which are slowly increasing and of exponential type. We equip this space with the classical topology.

The following theorem is given in [17, page 27]. 
Theorem 4.1. The transform $\mathcal{F}_{D}$ is a topological isomorphism

i) from $\mathcal{S}^{\prime}\left(\mathbb{R}^{d}\right)$ onto itself;

ii) from $\mathcal{E}^{\prime}\left(\mathbb{R}^{d}\right)$ onto $\mathcal{H}\left(\mathbb{C}^{d}\right)$.

Theorem 4.2. Let $S$ be in $\mathcal{S}^{\prime}\left(\mathbb{R}^{d}\right)$ and $\varphi$ in $\mathcal{S}\left(\mathbb{R}^{d}\right)$. Then, the distribution on $\mathbb{R}^{d}$ given by $\left(S *_{D} \varphi\right) \omega_{k}$ belongs to $\mathcal{S}^{\prime}\left(\mathbb{R}^{d}\right)$ and we have

$$
\mathcal{F}_{D}\left(T_{\left(S *_{D} \varphi\right) \omega_{k}}\right)=\mathcal{F}_{D}(\varphi) \mathcal{F}_{D}(S)
$$

Proof. i) As $S$ belongs to $\mathcal{S}^{\prime}\left(\mathbb{R}^{d}\right)$ then there exists a positive constant $C_{0}$ and $k_{0}, \ell_{0} \in \mathbb{N}$ such that

$$
\left|S *_{D} \varphi(x)\right|=\left|\left\langle S_{y}, \tau_{x} \varphi(-y)\right\rangle\right| \leq C_{0} Q_{k_{0}, \ell_{0}}\left(\tau_{x} \varphi\right) .
$$

But by using the inequality

$$
\forall x, y \in \mathbb{R}^{d}, \quad 1+\|x+y\|^{2} \leq 2\left(1+\|x\|^{2}\right)\left(1+\|y\|^{2}\right),
$$

the relations (4.2), (1.3) and the properties of the operator ${ }^{t} V_{k}$ (see Theorem 3.2 of [17]), we deduce that there exists a positive constant $C_{1}$ and $k, \ell \in \mathbb{N}$ such that

$$
Q_{\ell_{0}, \ell_{0}}\left(\tau_{x} \varphi\right) \leq C_{1}\left(1+\|x\|^{2}\right)^{\ell_{0}} Q_{k, \ell}(\varphi) .
$$

Thus from (4.7) we obtain

$$
\left|S *_{D} \varphi(x)\right| \leq C\left(1+\|x\|^{2}\right)^{\ell_{0}} Q_{k, \ell}(\varphi),
$$

where $C$ is a positive constant. This inequality shows that the distribution on $\mathbb{R}^{d}$ associated with the function $\left(S *_{D} \varphi\right) \omega_{k}$ belongs to $\mathcal{S}^{\prime}\left(\mathbb{R}^{d}\right)$.

ii) Let $\psi$ be in $\mathcal{S}\left(\mathbb{R}^{d}\right)$. We shall prove first that

$$
\left\langle T_{\left(S *_{D} \varphi\right) \omega_{k}}, \psi\right\rangle=\left\langle\check{S}, \varphi *_{D} \check{\psi}\right\rangle,
$$

where $\check{S}$ is the distribution in $\mathcal{S}^{\prime}\left(\mathbb{R}^{d}\right)$ given by

$$
\langle\check{S}, \phi\rangle=\langle S, \check{\phi}\rangle,
$$

with

$$
\forall x \in \mathbb{R}^{d}, \quad \check{\phi}(x)=\phi(-x) .
$$

We consider the two sequences $\left\{\varphi_{n}\right\}_{n \in \mathbb{N}}$ and $\left\{\psi_{m}\right\}_{m \in \mathbb{N}}$ in $\mathcal{D}\left(\mathbb{R}^{d}\right)$ which converge respectively to $\varphi$ and $\psi$ in $\mathcal{S}\left(\mathbb{R}^{d}\right)$. We have

$$
\begin{aligned}
\left\langle T_{\left(S *_{D} \varphi_{n}\right) \omega_{k}}, \psi_{m}\right\rangle & =\int_{\mathbb{R}^{d}}\left\langle S_{y}, \tau_{x} \varphi_{n}(-y)\right\rangle \psi_{m}(x) \omega_{k}(x) d x, \\
& =\left\langle S_{y}, \int_{\mathbb{R}^{d}} \psi_{m}(x) \tau_{x} \varphi_{n}(-y) \omega_{k}(x) d x\right\rangle=\left\langle S_{y}, \int_{\mathbb{R}^{d}} \check{\psi}_{m}(x) \tau_{-x} \varphi_{n}(-y) \omega_{k}(x) d x\right\rangle .
\end{aligned}
$$

Thus

$$
\left\langle T_{\left(S *_{D} \varphi_{n}\right) \omega_{k}}, \psi_{m}\right\rangle=\left\langle\check{S}, \varphi_{n} *_{D} \check{\psi}_{m}\right\rangle
$$


But

$$
\left\langle T_{\left(S *_{D} \varphi_{n}\right) \omega_{k}}, \psi_{m}\right\rangle-\left\langle T_{\left(S *_{D} \varphi\right) \omega_{k}}, \psi_{m}\right\rangle=\int_{\mathbb{R}^{d}} \check{S} *_{D}\left(\varphi_{n}-\varphi\right)(x) \check{\psi}_{m}(x) \omega_{k}(x) d x .
$$

Thus from (4.8) there exist a positive constant $M$ and $k, \ell \in \mathbb{N}$ such that

$$
\left|T_{\left(S *_{D} \varphi_{n}\right) \omega_{k}}, \psi_{m}\right\rangle-\left\langle T_{\left(S *_{D} \varphi\right) \omega_{k}}, \psi_{m}\right\rangle \mid \leq M Q_{k, \ell}\left(\varphi_{n}-\varphi\right) .
$$

Thus

$$
\left\langle T_{\left(S *_{D} \varphi_{n}\right) \omega_{k}}, \psi_{m}\right\rangle \underset{n \rightarrow+\infty}{\longrightarrow}\left\langle T_{\left(S *_{D} \varphi\right) \omega_{k}}, \psi_{m}\right\rangle .
$$

On the other hand we have

$$
\left\langle T_{\left(S *_{D} \varphi\right) \omega_{k}}, \psi_{m}\right\rangle \underset{m \rightarrow+\infty}{\longrightarrow}\left\langle T_{\left(S *_{D} \varphi\right) \omega_{k}}, \psi\right\rangle,
$$

and

$$
\varphi_{n} *_{D} \check{\psi}_{m} \underset{\substack{n \rightarrow+\infty \\ m \rightarrow+\infty}}{\longrightarrow} \varphi *_{D} \check{\psi}
$$

the limit is in $\mathcal{S}\left(\mathbb{R}^{d}\right)$.

We deduce (4.9) from (4.10), (4.11), (4.12) and (4.13).

We prove now the relation (4.6). Using (4.9) we obtain for all $\psi$ in $\mathcal{S}\left(\mathbb{R}^{d}\right)$

$$
\left\langle\mathcal{F}_{D}\left(T_{\left(S *_{D} \varphi\right) \omega_{k}}\right), \psi\right\rangle=\left\langle T_{\left(S *_{D} \varphi\right) \omega_{k}}, \mathcal{F}_{D}(\psi)\right\rangle,=\left\langle\check{S}, \varphi *_{D}\left(\mathcal{F}_{D}(\psi)\right)\right\rangle .
$$

But

$$
\varphi *_{D}\left(\mathcal{F}_{D}(\psi)\right)^{\swarrow}=\left(\mathcal{F}_{D}\left[\mathcal{F}_{D}(\varphi) \psi\right]\right) .
$$

Thus

$$
\left\langle\breve{S}, \varphi *_{D}\left(\mathcal{F}_{D}(\psi)\right)^{\dagger}\right\rangle=\left\langle S, \mathcal{F}_{D}\left[\mathcal{F}_{D}(\varphi) \psi\right]\right\rangle,=\left\langle\mathcal{F}_{D}(\varphi) \mathcal{F}_{D}(S), \psi\right\rangle
$$

Then

$$
\left\langle\mathcal{F}_{D}\left(T_{\left(S *_{D} \varphi\right) \omega_{k}}\right), \psi\right\rangle=\left\langle\mathcal{F}_{D}(\varphi) \mathcal{F}_{D}(S), \psi\right\rangle .
$$

This completes the proof of (4.6).

We consider the positive function $\varphi$ in $\mathcal{D}\left(\mathbb{R}^{d}\right)$ which is radial for $d \geq 2$ and even for $d=1$, with support in the closed ball of center 0 and radius 1 , satisfying

$$
\int_{\mathbb{R}^{d}} \varphi(x) \omega_{k}(x) d x=1,
$$

and $\phi$ the function on $[0,+\infty[$ given by

$$
\varphi(x)=\phi(\|x\|)=\phi(r) \quad \text { with } \quad r=\|x\| .
$$

For $\varepsilon \in] 0,1]$, we denote by $\varphi_{\varepsilon}$ the function on $\mathbb{R}^{d}$ defined by

$$
\forall x \in \mathbb{R}^{d}, \quad \varphi_{\varepsilon}(x)=\frac{1}{\varepsilon^{2 \gamma+d}} \phi\left(\frac{\|x\|}{\varepsilon}\right) .
$$

This function satisfies the following properties: 
i) Its support is contained in the closed ball $B_{\varepsilon}$ of center 0 , and radius $\varepsilon$.

ii) From [13, pages 585-586] we have

$$
\forall y \in \mathbb{R}^{d}, \quad \mathcal{F}_{D}\left(\varphi_{\varepsilon}\right)(y)=\frac{2^{\gamma+\frac{d}{2}}}{c_{k}} \mathcal{F}_{B}^{\gamma+\frac{d}{2}-1}(\phi)(\varepsilon\|y\|),
$$

where $\mathcal{F}_{B}^{\gamma+\frac{d}{2}-1}(f)(\lambda)$ is the Fourier-Bessel transform given by

$$
\forall \lambda \in \mathbb{R}, \quad \mathcal{F}_{B}^{\gamma+\frac{d}{2}-1}(f)(\lambda)=\int_{0}^{\infty} f(r) j_{\gamma+\frac{d}{2}-1}(\lambda r) \frac{r^{2 \gamma+d-1}}{2^{\gamma+\frac{d}{2}} \Gamma\left(\gamma+\frac{d}{2}\right)} d r,
$$

with $j_{\gamma+\frac{d}{2}-1}(\lambda r)$ the normalized Bessel function.

iii) There exists a positive constant $M$ such that

$$
\forall y \in \mathbb{R}^{d}, \quad\left|\mathcal{F}_{D}\left(\varphi_{\varepsilon}\right)(y)-1\right| \leq \varepsilon M\|y\|^{2} .
$$

Theorem 4.3. Let $S$ be in $\mathcal{S}^{\prime}\left(\mathbb{R}^{d}\right)$. We have

$$
\lim _{\varepsilon \rightarrow 0}\left(S *_{D} \phi_{\varepsilon}\right) \omega_{k}=S,
$$

where the limit is in $\mathcal{S}^{\prime}\left(\mathbb{R}^{d}\right)$.

Proof. We deduce (4.18) from (4.6), (4.15), (4.17) and Theorem 4.1.

Definition 4.3. Let $S_{1}$ be in $\mathcal{S}^{\prime}\left(\mathbb{R}^{d}\right)$ and $S_{2}$ in $\mathcal{E}^{\prime}\left(\mathbb{R}^{d}\right)$. The Dunkl convolution product of $S_{1}$ and $S_{2}$ is the distribution $S_{1} *_{D} S_{2}$ on $\mathbb{R}^{d}$ defined by

$$
\left\langle S_{1} *_{D} S_{2}, \psi\right\rangle=\left\langle S_{1, x},\left\langle S_{2, y}, \tau_{x} \psi(y)\right\rangle\right\rangle, \quad \psi \in \mathcal{D}\left(\mathbb{R}^{d}\right) .
$$

Remark 4.2. The relation (4.19) can also be written in the form

$$
\left\langle S_{1} *_{D} S_{2}, \psi\right\rangle=\left\langle S_{1}, \check{S}_{2} *_{D} \psi\right\rangle .
$$

Theorem 4.4. Let $S_{1}$ be in $\mathcal{S}^{\prime}\left(\mathbb{R}^{d}\right)$ and $S_{2}$ in $\mathcal{E}^{\prime}\left(\mathbb{R}^{d}\right)$. Then the distribution $S_{1} *_{D} S_{2}$ belongs to $\mathcal{S}^{\prime}\left(\mathbb{R}^{d}\right)$ and we have

$$
\mathcal{F}_{D}\left(S_{1} *_{D} S_{2}\right)=\mathcal{F}_{D}\left(S_{2}\right) \cdot \mathcal{F}_{D}\left(S_{1}\right)
$$

Proof. We deduce the result from (4.20), the relation

$$
T_{\left(\breve{S}_{2} *_{D} \mathcal{F}_{D}(\psi)\right) \omega_{k}}=\check{S}_{2} *_{D} T_{\mathcal{F}_{D}(\psi) \omega_{k}},
$$

and Theorem 4.2.

\subsection{Another proof of the geometrical form of the Paley-Wiener-Schwartz theorem for the Dunkl transform}

In this subsection we suppose that the multiplicity function satisfies $k(\alpha) \in \mathbb{N} \backslash\{0\}$ for all $\alpha \in R_{+}$.

The main result is to give another proof of the geometrical form of Paley-Wiener-Schwartz theorem for the transform $\mathcal{F}_{D}$, given in [17, pages 23-33]. 
Theorem 4.5. Let $E$ be a $W$-invariant compact convex set of $\mathbb{R}^{d}$ and $f$ an entire function on $\mathbb{C}^{d}$. Then $f$ is the Dunkl transform of a distribution in $\mathcal{E}^{\prime}\left(\mathbb{R}^{d}\right)$ with support in $E$ if and only if there exist a positive constant $C$ and $N \in \mathbb{N}$ such that

$$
\forall z \in \mathbb{C}^{d}, \quad|f(z)| \leq C\left(1+\|z\|^{2}\right)^{N} e^{I_{E}(\operatorname{Im} z)},
$$

where $I_{E}$ is the function given by (3.4).

Proof. Necessity condition. We consider a distribution $S$ in $\mathcal{E}^{\prime}\left(\mathbb{R}^{d}\right)$ with support in $E$.

Let $\mathcal{X}$ be in $\mathcal{D}\left(\mathbb{R}^{d}\right)$ equal to 1 in a neighborhood of $E$, and $\theta$ in $\mathcal{E}(\mathbb{R})$ such that

$$
\theta(t)= \begin{cases}1, & \text { if } t \leq 1 \\ 0, & \text { if } t>2\end{cases}
$$

We put $\eta=\operatorname{Im} z, z \in \mathbb{C}^{d}$ and we take $\varepsilon>0$. We denote by $\psi_{z}$ the function defined on $\mathbb{R}^{d}$ by

$$
\psi_{z}(x)=\chi(x) K(-i x, z)|W|^{-1} \sum_{w \in W} \theta\left(\|z\|^{\varepsilon}\left(\langle w x, \eta\rangle-I_{E}(\eta)\right)\right) .
$$

This function belongs to $\mathcal{D}\left(\mathbb{R}^{d}\right)$ and as $E$ is $W$-invariant, then it is equal to $K(-i x, z)$ in a neighborhood of $E$. Thus

$$
\forall z \in \mathbb{C}^{d}, \quad \mathcal{F}_{D}(S)(z)=\left\langle S_{x}, \psi_{z}(x)\right\rangle .
$$

As $S$ is with compact support, then it is of finite order $N$. Then there exists a positive constant $C_{0}$ such that

$$
\forall z \in \mathbb{C}^{d}, \quad\left|\mathcal{F}_{D}(S)(z)\right| \leq C_{0} \sum_{|p| \leq N} \sup _{x \in \mathbb{R}^{d}}\left|D^{p} \psi_{z}(x)\right| .
$$

Using the Leibniz rule, we obtain

$$
\begin{aligned}
\forall x \in \mathbb{R}^{d}, \quad D^{p} \psi_{z}(x)= & \sum_{q+r+s=p} \frac{p !}{q ! r ! s !} D^{q} \mathcal{X}(x) D^{r} K(-i x, z) \\
& \times D^{s}|W|^{-1} \sum_{w \in W} \theta\left(\|z\|^{\varepsilon}\left(\langle w x, \eta\rangle-I_{E}(\eta)\right)\right) .
\end{aligned}
$$

We have

$$
\forall x \in \mathbb{R}^{d}, \quad\left|D^{q} \chi(x)\right| \leq \text { const }
$$

and if $M$ is the estimate of $\sup _{t \in \mathbb{R}}\left|\theta^{(k)}(t)\right|, k \leq N$, we obtain

$$
\forall x \in \mathbb{R}^{d}, \quad\left|D^{s}\left(\sum_{w \in W} \theta\left(\|z\|^{\varepsilon}\left(\langle w x, \eta\rangle-I_{E}(\eta)\right)\right)\right)\right| \leq M\left(\|z\|^{\varepsilon}\|\eta\|\right)^{|s|} .
$$

On the other hand from (2.3) we have

$$
\forall x \in \mathbb{R}^{d}, \quad\left|D^{r} K(-i x, z)\right| \leq\|z\|^{r} e^{\max _{w \in W}\langle w x, \eta\rangle} .
$$

Using inequalities (4.24), (4.25), (4.26) and (4.23) we deduce that there exists a positive constant $C_{1}$ such that

$$
\forall x \in \mathbb{R}^{d}, \quad\left|D^{p} \psi_{z}(x)\right| \leq C_{1}\left(1+\|z\|^{2}\right)^{N(1+\varepsilon)} e^{\max _{w \in W}\langle w x, \eta\rangle} .
$$


From this relation and (4.22) we obtain

$$
\forall z \in \mathbb{C}^{d}, \quad\left|\mathcal{F}_{D}(S)(z)\right| \leq C_{2}\left(1+\|z\|^{2}\right)^{N(1+\varepsilon)} \sup _{x \in E} e^{\max _{w \in W}\langle w x, \eta\rangle},
$$

where $C_{2}$ is a positive constant, and the supremum is calculated when $\|z\| \geq 1$, for

$$
\langle w x, \eta\rangle \leq I_{E}(\eta)+\frac{2}{\|z\|^{\varepsilon}}
$$

because if not we have $\theta=0$. This inequality implies

$$
\sup _{x \in E} e^{\max _{w \in W}\langle w x, \eta\rangle} \leq e^{2} \cdot e^{I_{E}(\eta)}
$$

From $(4.27),(4.28)$ we deduce that there exists a positive constant $C_{3}$ independent from $\varepsilon$ such that

$$
\forall z \in \mathbb{C}^{d}, \quad\|z\| \geq 1, \quad\left|\mathcal{F}_{D}(S)(z)\right| \leq C_{3}\left(1+\|z\|^{2}\right)^{N(1+\varepsilon)} e^{I_{E}(\eta)} .
$$

If we make $\varepsilon \rightarrow 0$ in this relation we obtain (4.21) for $\|z\| \geq 1$. But this inequality is also true (with another constant) for $\|z\| \leq 1$, because in the set $\left\{z \in \mathbb{C}^{d},\|z\| \leq 1\right\}$ the function $\mathcal{F}_{D}(S)(z) e^{-I_{E}(\eta)}$ is bounded.

Sufficient condition. Let $f$ be an entire function on $\mathbb{C}^{d}$ satisfying the condition (4.21). It is clear that the distribution given by the restriction of $f \omega_{k}$ to $\mathbb{R}^{d}$ belongs to $\mathcal{S}^{\prime}\left(\mathbb{R}^{d}\right)$. Thus from Theorem 4.1i there exists a distribution $S$ in $\mathcal{S}^{\prime}\left(\mathbb{R}^{d}\right)$ such that

$$
T_{f \omega_{k}}=\mathcal{F}_{D}(S)
$$

We shall show that the support of $S$ is contained in $E$. Let $\varphi_{\varepsilon}$ be the function given by the relation (4.14). We consider the distribution

$$
T_{f_{\varepsilon} \omega_{k}}=\mathcal{F}_{D}\left(T_{\left(S *_{D} \varphi_{\varepsilon}\right) \omega_{k}}\right) .
$$

From Theorem 4.2 and (4.29), (4.30) we deduce that

$$
f_{\varepsilon}=\mathcal{F}_{D}\left(\varphi_{\varepsilon}\right) f .
$$

The properties of the function $f$ and (4.15), (4.16) and (4.17) show that the function $f_{\varepsilon}$ can be extended to an entire function on $\mathbb{C}^{d}$ which satisfies: for all $q \in \mathbb{N}$ there exists a positive constant $C_{q}$ such that

$$
\forall z \in \mathbb{C}^{d}, \quad\left|f_{\varepsilon}(z)\right| \leq C_{q}(1+\|z\|)^{-q} e^{I_{E+B_{\varepsilon}}(\operatorname{Im} z)} .
$$

Then from (4.31), Theorem 3.2 and (4.30), the function $\left(S * \varphi_{\varepsilon}\right) \omega_{k}$ belongs to $\mathcal{D}\left(\mathbb{R}^{d}\right)$ with support in $E+B_{\varepsilon}$. But from Theorem 4.3, the family $\left(S * \varphi_{\varepsilon}\right) \omega_{k}$ converges to $S$ in $\mathcal{S}^{\prime}\left(\mathbb{R}^{d}\right)$ when $\varepsilon$ tends to zero. Thus for all $\varepsilon>0$, the support of $S$ is in $E+B_{\varepsilon}$, then it is contained in $E$.

Remark 4.3. In the following we give an ameliorated version of the proof of Proposition 6.3 of [17, page 30$]$.

Let $E$ be a $W$-invariant compact convex set of $\mathbb{R}^{d}$ and $x \in E$. The function $f(x, \cdot)$ defined on $\mathbb{C}^{d}$ by

$$
f(x, z)=e^{-i\left(\sum_{j=1}^{d} x_{j} z_{j}\right)},
$$


is entire on $\mathbb{C}^{d}$ and satisfies

$$
\forall z \in \mathbb{C}^{d}, \quad|f(x, z)| \leq e^{I_{E}(\operatorname{Im} z)} .
$$

Thus from Theorem 4.5 there exists a distribution $\tilde{\eta}_{x}$ in $\mathcal{E}^{\prime}\left(\mathbb{R}^{d}\right)$ with support in $E$ such that

$$
\forall y \in \mathbb{R}^{d}, \quad f(x, y)=e^{-i\langle x, y\rangle}=\left\langle\tilde{\eta}_{x}, K(-i y, \cdot)\right\rangle .
$$

Applying now the remainder of the proof given in [17, page 32], we deduce that the support

of the representing distribution $\eta_{x}$ of the inverse Dunkl intertwining operator $V_{k}^{-1}$ is contained in $E$.

\section{Inversion formulas for the Dunkl intertwining operator and its dual}

\subsection{The pseudo-differential operators $P$}

Definition 5.1. We define the pseudo-differential operator $P$ on $\mathcal{S}\left(\mathbb{R}^{d}\right)$ by

$$
\forall x \in \mathbb{R}^{d}, \quad P(f)(x)=\frac{\pi^{d} c_{k}^{2}}{2^{2 \gamma}} \mathcal{F}^{-1}\left[\omega_{k} \mathcal{F}(f)\right](x) .
$$

Proposition 5.1. The distribution $T_{\omega_{k}}$ given by the function $\omega_{k}$, is in $\mathcal{S}^{\prime}\left(\mathbb{R}^{d}\right)$ and for all $f$ in $\mathcal{S}\left(\mathbb{R}^{d}\right)$ we have

$$
\forall x \in \mathbb{R}^{d}, \quad P(f)(x)=\frac{\pi^{d} c_{k}^{2}}{2^{2 \gamma}} \mathcal{F}\left(T_{\omega_{k}}\right) * \breve{f}(-x) .
$$

where $*$ is the classical convolution production of a distribution and a function on $\mathbb{R}^{d}$.

Proof. It is clear that the distribution $T_{\omega_{k}}$ given by the function $\omega_{k}$ belongs to $\mathcal{S}^{\prime}\left(\mathbb{R}^{d}\right)$. On the other hand from the relation (5.1) we have

$$
\forall x \in \mathbb{R}^{d}, \quad P(f)(x)=\frac{\pi^{d} c_{k}^{2}}{2^{2 \gamma}} \int_{\mathbb{R}^{d}} \mathcal{F}(f(\xi+x))(y) \omega_{k}(y) d y
$$

Thus

$$
\forall x \in \mathbb{R}^{d}, \quad P(f)(x)=\frac{\pi^{d} c_{k}^{2}}{2^{2 \gamma}}\left\langle\mathcal{F}\left(T_{\omega_{k}}\right)_{y}, f(x+y)\right\rangle .
$$

With the definition of the classical convolution product of a distribution and a function on $\mathbb{R}^{d}$, the relation (5.2) can also be written in the form

$$
\forall x \in \mathbb{R}^{d}, \quad P(f)(x)=\frac{\pi^{d} c_{k}^{2}}{2^{2 \gamma}} \mathcal{F}\left(T_{\omega_{k}}\right) * \breve{f}(-x) .
$$

Proposition 5.2. For all $f$ in $\mathcal{S}\left(\mathbb{R}^{d}\right)$ the function $P(f)$ is of class $C^{\infty}$ on $\mathbb{R}^{d}$ and we have

$$
\forall x \in \mathbb{R}^{d}, \quad \frac{\partial}{\partial x_{j}} P(f)(x)=P\left(\frac{\partial}{\partial \xi_{j}} f\right)(x), \quad j=1,2, \ldots, d .
$$

Proof. By derivation under the integral sign, and by using the relation

$$
\forall y \in \mathbb{R}^{d}, \quad i y_{j} \mathcal{F}(f)(y)=\mathcal{F}\left(\frac{\partial}{\partial \xi_{j}} f\right)(y),
$$

we obtain (5.3). 


\subsection{Inversion formulas for the Dunkl intertwining operator and its dual on the space $\mathcal{S}\left(\mathbb{R}^{d}\right)$}

Theorem 5.1. For all $f$ in $\mathcal{S}\left(\mathbb{R}^{d}\right)$ we have

$$
\forall x \in \mathbb{R}^{d}, \quad{ }^{t} V_{k}^{-1}(f)(x)=V_{k}(P(f))(x) .
$$

Proof. From [15, Theorem 4.1] for all $f$ in $\mathcal{S}\left(\mathbb{R}^{d}\right)$, the function ${ }^{t} V_{k}^{-1}(f)$ belongs to $\mathcal{S}\left(\mathbb{R}^{d}\right)$. Then from Theorem 3.1 we have

$$
\forall x \in \mathbb{R}^{d}, \quad{ }^{t} V_{k}^{-1}(f)(x)=\frac{c_{k}^{2}}{2^{2 \gamma+d}} \int_{\mathbb{R}^{d}} K(i y, x) \mathcal{F}_{D}\left({ }^{t} V_{k}^{-1}(f)\right)(y) \omega_{k}(y) d y .
$$

But from the relations (3.2), (1.7), (1.3), we have

$$
\forall y \in \mathbb{R}^{d}, \quad \mathcal{F}_{D}\left({ }^{t} V_{k}^{-1}(f)\right)(y)=\mathcal{F}(f)(y),
$$

and

$$
\forall y \in \mathbb{R}^{d}, \quad K(i y, x)=\mathcal{F}\left(\breve{\mu}_{x}\right)(y),
$$

where $\breve{\mu}_{x}$ is the probability measure given for a continuous function $f$ on $\mathbb{R}^{d}$ by

$$
\int_{\mathbb{R}^{d}} f(t) d \check{\mu}_{x}(t)=\int_{\mathbb{R}^{d}} f(-t) d \mu_{x}(t) .
$$

Thus (5.5) can also be written in the form

$$
\forall x \in \mathbb{R}^{d}, \quad{ }^{t} V_{k}^{-1}(f)(x)=\frac{c_{k}^{2}}{2^{2 \gamma+d}} \int_{\mathbb{R}} \mathcal{F}\left(\breve{\mu}_{x}\right)(y) \omega_{k}(y) \mathcal{F}(f)(y) d y .
$$

Then by using (5.1), the properties of the Fourier transform $\mathcal{F}$ and Fubini's theorem we obtain

$$
\forall x \in \mathbb{R}^{d}, \quad{ }^{t} V_{k}^{-1}(f)(x)=\frac{c_{k}^{2}}{2^{2 \gamma+d}} \int_{\mathbb{R}^{d}} \mathcal{F}\left[\omega_{k} \mathcal{F}(f)\right](y) d \breve{\mu}_{x}(y)=\int_{\mathbb{R}^{d}} P(f)(y) d \mu_{x}(y) .
$$

Thus

$$
\forall x \in \mathbb{R}^{d}, \quad{ }^{t} V_{k}^{-1}(f)(x)=V_{k}(P(f))(x) .
$$

Theorem 5.2. For all $f$ in $\mathcal{S}\left(\mathbb{R}^{d}\right)$ we have

$$
\forall x \in \mathbb{R}^{d}, \quad V_{k}^{-1}(f)(x)=P^{t} V_{k}(f)(x) .
$$

Proof. We deduce the relation (5.6) by replacing $f$ by ${ }^{t} V_{k}(f)$ in (5.4) and by using the fact that the operator $V_{k}$ is an isomorphism from $\mathcal{E}\left(\mathbb{R}^{d}\right)$ onto itself.

\subsection{Inversion formulas for the dual Dunkl intertwining operator on the space $\mathcal{E}^{\prime}\left(\mathbb{R}^{d}\right)$}

The dual Dunkl intertwining operator ${ }^{t} V_{k}$ on $\mathcal{E}^{\prime}\left(\mathbb{R}^{d}\right)$ is defined by

$$
\left\langle{ }^{t} V_{k}(S), f\right\rangle=\left\langle S, V_{k}(f)\right\rangle, \quad f \in \mathcal{E}\left(\mathbb{R}^{d}\right) .
$$

The operator ${ }^{t} V_{k}$ is a topological isomorphism from $\mathcal{E}^{\prime}\left(\mathbb{R}^{d}\right)$ onto itself. The inverse operator is given by

$$
\left\langle{ }^{t} V_{k}^{-1}(S), f\right\rangle=\left\langle S, V_{k}^{-1}(f)\right\rangle, \quad f \in \mathcal{E}\left(\mathbb{R}^{d}\right),
$$

see [17, pages $26-27]$.

Theorem 5.3. For all $S$ in $\mathcal{E}^{\prime}\left(\mathbb{R}^{d}\right)$ the operator ${ }^{t} V_{k}^{-1}$ satisfies also the relation

$$
\left\langle{ }^{t} V_{k}^{-1}(S), f\right\rangle=\left\langle S, P^{t} V_{k}(f)\right\rangle, \quad f \in \mathcal{S}\left(\mathbb{R}^{d}\right) .
$$

Proof. We deduce (5.8) from (5.6) and (5.7). 


\section{Other expressions of the inversion formulas for the Dunkl intertwining operator and its dual when the multiplicity function is integer}

In this section we suppose that the multiplicity function satisfies $k(\alpha) \in \mathbb{N} \backslash\{0\}$ for all $\alpha \in R_{+}$. The following two Propositions give some other properties of the operator $P$ defined by (5.1).

Proposition 6.1. Let $E$ be a compact convex set of $\mathbb{R}^{d}$. Then for all $f$ in $\mathcal{D}\left(\mathbb{R}^{d}\right)$ we have $\operatorname{supp} f \subset E \Rightarrow \operatorname{supp} P(f) \subset E$.

Proof. From the relation (5.1) we have

$$
\forall x \in \mathbb{R}^{d}, \quad P(f)(x)=\frac{\pi^{d} c_{k}^{2}}{2^{2 \gamma}} \int_{\mathbb{R}^{d}} \mathcal{F} f(y) e^{i\langle x, y\rangle} \omega_{k}(y) d y .
$$

We consider the function $F$ defined by

$$
\forall z \in \mathbb{C}^{d}, \quad F(z)=\left(\prod_{\alpha \in R_{+}}(\langle\alpha, z\rangle)^{2 k(\alpha)}\right) \mathcal{F}(f)(z) .
$$

This function is entire on $\mathbb{C}^{d}$ and by using Theorem 2.6 of [1] we deduce that for all $q \in \mathbb{N}$, there exists a positive constant $C_{q}$ such that

$$
\forall z \in \mathbb{C}^{d}, \quad|F(z)| \leq C_{q}\left(1+\|z\|^{2}\right)^{-q} e^{I_{E}(\operatorname{Im} z)},
$$

where $I_{E}$ is the function given by (3.4).

The relation (6.1) can also be written in the form

$$
\forall x \in \mathbb{R}^{d}, \quad P(f)(x)=\frac{\pi^{d} c_{k}^{2}}{2^{2 \gamma}} \int_{\mathbb{R}^{d}} F(y) e^{i\langle x, y\rangle} d y .
$$

Thus (6.3), (6.2) and Theorem 2.6 of [1], imply that supp $P f \subset E$.

Proposition 6.2. For all $f$ in $\mathcal{S}\left(\mathbb{R}^{d}\right)$ we have

$$
P(f)=\frac{\pi^{d} c_{k}^{2}}{2^{2 \gamma}}\left[\prod_{\alpha \in R_{+}}(-1)^{k(\alpha)}\left(\alpha_{1} \frac{\partial}{\partial \xi_{1}}+\cdots+\alpha_{d} \frac{\partial}{\partial \xi_{d}}\right)^{2 k(\alpha)}\right](f) .
$$

Proof. For all $f$ in $\mathcal{S}\left(\mathbb{R}^{d}\right)$, we have

$$
\forall y \in \mathbb{R}^{d}, \quad \omega_{k}(y) \mathcal{F}(f)(y)=\prod_{\alpha \in R_{+}}(\langle\alpha, y\rangle)^{2 k(\alpha)} \mathcal{F}(f)(y) .
$$

But

$$
\forall y \in \mathbb{R}^{d}, \quad\langle\alpha, y\rangle \mathcal{F}(f)(y)=\mathcal{F}\left[-i\left(\alpha_{1} \frac{\partial}{\partial \xi_{1}}+\cdots+\alpha_{d} \frac{\partial}{\partial \xi_{d}}\right) f\right](y) .
$$

From (6.5), (6.6) we obtain

$$
\forall y \in \mathbb{R}^{d}, \quad \omega_{k}(y) \mathcal{F}(f)(y)=\mathcal{F}\left[\prod_{\alpha \in R_{+}}(-1)^{k(\alpha)}\left(\alpha_{1} \frac{\partial}{\partial \xi_{1}}+\cdots+\alpha_{d} \frac{\partial}{\partial \xi_{d}}\right)^{2 k(\alpha)} f\right](y) .
$$

This relation, Definition 5.1 and the inversion formula for the Fourier transform $\mathcal{F}$ imply (6.4).

Remark 6.1. In this case the operator $P$ is not a pseudo-differential operator but it is a partial differential operator. 


\subsection{The differential-difference operator $Q$}

Definition 6.1. We define the differential-difference operator $Q$ on $\mathcal{S}\left(\mathbb{R}^{d}\right)$ by

$$
\forall x \in \mathbb{R}^{d}, \quad Q(f)(x)={ }^{t} V_{k}^{-1} \circ P \circ{ }^{t} V_{k}(f)(x) .
$$

\section{Proposition 6.3.}

i) The operator $Q$ is linear and continuous from $\mathcal{S}\left(\mathbb{R}^{d}\right)$ into itself.

ii) For all $f$ in $\mathcal{S}\left(\mathbb{R}^{d}\right)$ we have

$$
\forall x \in \mathbb{R}^{d}, \quad T_{j} Q(f)(x)=Q\left(T_{j} f\right)(x), \quad j=1, \ldots, d,
$$

where $T_{j}, j=1,2, \ldots, d$, are the Dunkl operators.

Proof. We deduce the result from the properties of the operator ${ }^{t} V_{k}$ (see Theorem 3.2 of [17]), and Proposition 5.2.

Proposition 6.4. For all $f$ in $\mathcal{S}\left(\mathbb{R}^{d}\right)$ we have

$$
\forall x \in \mathbb{R}^{d}, \quad Q(f)(x)=\frac{\pi^{d} c_{k}^{2}}{2^{2 \gamma}} \mathcal{F}_{D}^{-1}\left(\omega_{k} \mathcal{F}_{D}(f)\right)(x) .
$$

Proof. Using the relations (3.2), (5.1) and the properties of the operator ${ }^{t} V_{k}$ (see Theorem 3.2 of [17]), we deduce from Definition 6.1 that

$$
\forall x \in \mathbb{R}^{d}, \quad Q(f)(x)=\mathcal{F}_{D}^{-1}\left\{\mathcal{F} \circ P\left({ }^{t} V_{k}(f)\right)\right\}(x)=\frac{\pi^{d} c_{k}^{2}}{2^{2 \gamma}} \mathcal{F}_{D}^{-1}\left\{\mathcal{F} \circ \mathcal{F}^{-1}\left[\omega_{k} \mathcal{F}_{D}(f)\right]\right\}(x) .
$$

As the function $\omega_{k} \mathcal{F}_{D}(f)$ belongs to $\mathcal{S}\left(\mathbb{R}^{d}\right)$, then by applying the fact that the classical Fourier transform $\mathcal{F}$ is bijective from $\mathcal{S}\left(\mathbb{R}^{d}\right)$ onto itself, we obtain

$$
\forall x \in \mathbb{R}^{d}, \quad Q(f)(x)=\frac{\pi^{d} c_{k}^{2}}{2^{2 \gamma}} \mathcal{F}_{D}^{-1}\left(\omega_{k} \mathcal{F}_{D}(f)\right)(x) .
$$

Proposition 6.5. The distribution $T_{\omega_{k}^{2}}$ given by the function $\omega_{k}^{2}$ is in $\mathcal{S}^{\prime}\left(\mathbb{R}^{d}\right)$ and for all $f$ in $\mathcal{S}\left(\mathbb{R}^{d}\right)$ we have

$$
\forall x \in \mathbb{R}^{d}, \quad Q(f)(x)=\frac{\pi^{d} c_{k}^{4}}{2^{4 \gamma+d}} \mathcal{F}_{D}\left(T_{\omega_{k}^{2}}\right) *_{D} \breve{f}(-x),
$$

where $*_{D}$ is the Dunkl convolution product of a distribution and a function on $\mathbb{R}^{d}$.

Proof. It is clear that the distribution $T_{\omega_{k}^{2}}$ given by the function $\omega_{k}^{2}$ belongs to $\mathcal{S}^{\prime}\left(\mathbb{R}^{d}\right)$. On the other hand from the relations (6.7), (3.3) and (4.3) we obtain

$$
\begin{aligned}
\forall x \in \mathbb{R}^{d}, \quad Q(f)(x) & =\frac{\pi^{d} c_{k}^{4}}{2^{4 \gamma+d}} \int_{\mathbb{R}^{d}} \mathcal{F}_{D}\left(\tau_{x}(f)\right)(y) \omega_{k}^{2}(y) d y \\
& =\frac{\pi^{d} c_{k}^{4}}{2^{4 \gamma+d}}\left\langle\mathcal{F}\left(T_{\omega_{k}^{2}}\right)_{y}, \tau_{x}(f)(y)\right\rangle .
\end{aligned}
$$

Thus Definition 4.1 implies

$$
\forall x \in \mathbb{R}^{d}, \quad Q(f)(x)=\frac{\pi^{d} c_{k}^{4}}{2^{4 \gamma+d}} \mathcal{F}_{D}\left(T_{\omega_{k}^{2}}\right) *_{D} \breve{f}(-x) .
$$


Proposition 6.6. For all $f$ in $\mathcal{S}\left(\mathbb{R}^{d}\right)$ we have

$$
Q(f)=\frac{\pi^{d} c_{k}^{2}}{2^{2 \gamma}}\left[\prod_{\alpha \in R_{+}}(-1)^{k(\alpha)}\left(\alpha_{1} T_{1}+\cdots+\alpha_{d} T_{d}\right)^{2 k(\alpha)}\right](f) .
$$

Proof. For all $f$ in $\mathcal{S}\left(\mathbb{R}^{d}\right)$, we have

$$
\forall y \in \mathbb{R}^{d}, \quad \omega_{k}(y) \mathcal{F}_{D}(f)(y)=\prod_{\alpha \in R_{+}}(\langle\alpha, y\rangle)^{2 k(\alpha)} \mathcal{F}_{D}(f)(y) .
$$

But using (2.1), (2.2) we deduce that

$$
\forall y \in \mathbb{R}^{d}, \quad\langle\alpha, y\rangle \mathcal{F}_{D}(f)(y)=\mathcal{F}_{D}\left[-i\left(\alpha_{1} T_{1}+\cdots+\alpha_{d} T_{d}\right) f\right](y) .
$$

From (6.9), (6.10) we obtain

$$
\forall y \in \mathbb{R}^{d}, \quad \omega_{k}(y) \mathcal{F}_{D}(f)(y)=\mathcal{F}_{D}\left[\prod_{\alpha \in R_{+}}(-1)^{k(\alpha)}\left(\alpha_{1} T_{1}+\cdots+\alpha_{d} T_{d}\right)^{2 k(\alpha)} f\right](y) .
$$

This relation, Propositions 6.3, 6.4 and Theorem 3.1 imply (6.8).

\subsection{Other expressions of the inversion formulas for the Dunkl intertwining operator and its dual on spaces of functions and distributions}

In this subsection we give other expressions of the inversion formulas for the operators $V_{k}$ and ${ }^{t} V_{k}$ and we deduce the expressions of the representing distributions of the operators $V_{k}^{-1}$ and ${ }^{t} V_{k}^{-1}$.

Theorem 6.1. For all $f$ in $\mathcal{S}\left(\mathbb{R}^{d}\right)$ we have

$$
\forall x \in \mathbb{R}^{d}, \quad V_{k}^{-1}(f)(x)={ }^{t} V_{k}(Q(f))(x) .
$$

Proof. We obtain this result by using of Proposition 6.3, Theorem 5.2 and Definition 6.1.

Proposition 6.7. Let $E$ be a $W$-invariant compact convex set of $\mathbb{R}^{d}$. Then for all $f$ in $\mathcal{D}\left(\mathbb{R}^{d}\right)$ we have

$$
\operatorname{supp} f \subset E \Longleftrightarrow \operatorname{supp}^{t} V_{k}(f) \subset E \text {. }
$$

Proof. For all $f$ in $\mathcal{D}\left(\mathbb{R}^{d}\right)$, we obtain from (3.2) the relations

$$
\begin{aligned}
& { }^{t} V_{k}(f)=\mathcal{F}^{-1} \circ \mathcal{F}_{D}(f), \\
& { }^{t} V_{k}^{-1}(f)=\mathcal{F}_{D}^{-1} \circ \mathcal{F}(f) .
\end{aligned}
$$

We deduce (6.12) from these relations, Theorem 3.2 and Theorem 2.6 of [1].

Proposition 6.8. Let $E$ be a $W$-invariant compact convex set of $\mathbb{R}^{d}$. Then for all $f$ in $\mathcal{D}\left(\mathbb{R}^{d}\right)$ we have

$$
\operatorname{supp} f \subset E \Rightarrow \operatorname{supp} Q(f) \subset E \text {. }
$$

Proof. We obtain (6.13) from Definition 6.1, Propositions 6.1 and 6.7. 
Theorem 6.2. For all $S$ in $\mathcal{E}^{\prime}\left(\mathbb{R}^{d}\right)$ the operator ${ }^{t} V_{k}^{-1}$ satisfies also the relation

$$
\left\langle{ }^{t} V_{k}^{-1}(S), f\right\rangle=\left\langle S,{ }^{t} V_{k}(Q(f))\right\rangle, \quad f \in \mathcal{S}\left(\mathbb{R}^{d}\right) .
$$

Proof. We deduce (6.14) from (5.7) and (6.11).

Corollary 6.1. Let $E$ be a $W$-invariant compact convex set of $\mathbb{R}^{d}$. For all $S$ in $\mathcal{E}^{\prime}\left(\mathbb{R}^{d}\right)$ with $\operatorname{supp} S \subset E$, we have

$$
\operatorname{supp}^{t} V_{k}^{-1}(S) \subset E \text {. }
$$

Definition 6.2. We define the transposed operators ${ }^{t} P$ and ${ }^{t} Q$ of the operators $P$ and $Q$ on $\mathcal{S}^{\prime}\left(\mathbb{R}^{d}\right)$ by

$$
\begin{array}{ll}
\left\langle{ }^{t} P(S), f\right\rangle=\langle S, P(f)\rangle, & f \in \mathcal{S}\left(\mathbb{R}^{d}\right), \\
\left\langle{ }^{t} Q(S), f\right\rangle=\langle S, Q(f)\rangle, & f \in \mathcal{S}\left(\mathbb{R}^{d}\right) .
\end{array}
$$

Proposition 6.9. For all $S$ in $\mathcal{S}^{\prime}\left(\mathbb{R}^{d}\right)$ we have

$$
\begin{aligned}
& { }^{t} P(S)=\frac{\pi^{d} c_{k}^{2}}{2^{2 \gamma}}\left[\prod_{\alpha \in R_{+}}\left(\alpha \frac{\partial}{\partial \xi_{1}}+\cdots+\alpha_{d} \frac{\partial}{\partial \xi_{d}}\right)^{2 k(\alpha)}\right] S, \\
& { }^{t} Q(S)=\frac{\pi^{d} c_{k}^{2}}{2^{2 \gamma}}\left[\prod_{\alpha \in R_{+}}\left(\alpha T_{1}+\cdots+\alpha_{d} T_{d}\right)^{2 k(\alpha)}\right] S,
\end{aligned}
$$

where $T_{j}, j=1,2, \ldots, d$, are the Dunkl operators defined on $\mathcal{S}^{\prime}\left(\mathbb{R}^{d}\right)$ by

$$
\left\langle T_{j} S, f\right\rangle=-\left\langle S, T_{j} f\right\rangle, \quad f \in \mathcal{S}\left(\mathbb{R}^{d}\right) .
$$

Proposition 6.10. For all $S$ in $\mathcal{S}^{\prime}\left(\mathbb{R}^{d}\right)$ we have

$$
\begin{aligned}
& \mathcal{F}^{-1}\left({ }^{t} P(S)\right)=\frac{\pi^{d} c_{k}^{2}}{2^{2 \gamma}} \mathcal{F}^{-1}(S) \omega_{k}, \\
& \mathcal{F}_{D}^{-1}\left({ }^{t} Q(S)\right)=\frac{\pi^{d} c_{k}^{2}}{2^{2 \gamma}} \mathcal{F}_{D}^{-1}(S) \omega_{k} .
\end{aligned}
$$

Proof. We deduce these relations from (5.1), (6.7) and the definitions of the classical Fourier transform and the Dunkl transform of tempered distributions on $\mathbb{R}^{d}$.

Theorem 6.3. The representing distributions $\eta_{x}$ and $Z_{x}$ of the inverse of the Dunkl intertwining operator and its dual, are given by

$$
\forall x \in \mathbb{R}^{d}, \quad \eta_{x}={ }^{t} Q\left(\nu_{x}\right)
$$

and

$$
\forall x \in \mathbb{R}^{d}, \quad Z_{x}={ }^{t} P\left(\mu_{x}\right),
$$

where $\mu_{x}$ and $\nu_{x}$ are the representing measures of the Dunkl intertwining operator $V_{k}$ and its dual ${ }^{t} V_{k}$. 
Proof. From (1.5), for all $f$ in $\mathcal{S}\left(\mathbb{R}^{d}\right)$ we have

$$
\forall x \in \mathbb{R}^{d}, \quad{ }^{t} V_{k}(Q(f))(x)=\left\langle\nu_{x}, Q(f)\right\rangle=\left\langle{ }^{t} Q\left(\nu_{x}\right), f\right\rangle .
$$

On the other hand from (1.4)

$$
\forall x \in \mathbb{R}^{d}, \quad V_{k}^{-1}(f)(x)=\left\langle\eta_{x}, f\right\rangle
$$

We obtain (6.15) from this relation, (6.17) and (6.11).

Using (1.3), for all $f$ in $\mathcal{S}\left(\mathbb{R}^{d}\right)$ we can also write the relation (5.4) in the form

$$
\forall x \in \mathbb{R}^{d}, \quad{ }^{t} V_{k}^{-1}(f)(x)=\left\langle\mu_{x}, P(f)\right\rangle=\left\langle{ }^{t} P\left(\mu_{x}\right), f\right\rangle .
$$

But from (1.6) we have

$$
\forall x \in \mathbb{R}^{d}, \quad{ }^{t} V^{-1}(f)(x)=\left\langle Z_{x}, f\right\rangle .
$$

We deduce (6.16) from this relation and (6.18).

Corollary 6.2. We have

$$
\forall x \in \mathbb{R}^{d}, \quad \eta_{x}=\frac{\pi^{d} c_{k}^{2}}{2^{2 \gamma}}\left[\prod_{\alpha \in R_{+}}\left(\alpha_{1} T_{1}+\cdots+\alpha_{d} T_{d}\right)^{2 k(\alpha)}\right]\left(\nu_{x}\right)
$$

and

$$
\forall x \in \mathbb{R}^{d}, \quad Z_{x}=\frac{\pi^{d} c_{k}^{2}}{2^{2 \gamma}}\left[\prod_{\alpha \in R_{+}}\left(\alpha_{1} \frac{\partial}{\partial \xi_{1}}+\cdots+\alpha_{d} \frac{\partial}{\partial \xi_{d}}\right)^{2 k(\alpha)}\right]\left(\mu_{x}\right) .
$$

Proof. We deduce these relations from Theorem 6.3 and Proposition 6.9.

\section{Applications}

\subsection{Other proof of the sufficiency condition of Theorem 4.4}

Let $f$ be an entire function on $\mathbb{C}^{d}$ satisfying the condition (4.21). Then from Theorem 2.6 of [1], the distribution $\mathcal{F}^{-1}(f)$ belongs to $\mathcal{E}^{\prime}\left(\mathbb{R}^{d}\right)$ and we have

$$
\operatorname{supp} \mathcal{F}^{-1}(f) \subset E \text {. }
$$

From the relation

$$
\mathcal{F}_{D}^{-1}(f)={ }^{t} V_{k}^{-1} \circ \mathcal{F}^{-1}(f)
$$

given in [17, page 27] and Corollary 6.1, we deduce that the distribution $\mathcal{F}_{D}^{-1}(f)$ is in $\mathcal{E}^{\prime}\left(\mathbb{R}^{d}\right)$ and its support is contained in $E$. 


\subsection{Other expressions of the Dunkl translation operators}

We consider the Dunkl translation operators $\tau_{x}, x \in \mathbb{R}^{d}$, given by the relations (4.1), (4.2).

\section{Theorem 7.1.}

i) When the multiplicity function $k(\alpha)$ satisfies $k(\alpha)>0$ for all $\alpha \in R_{+}$, we have

$$
\forall y \in \mathbb{R}^{d}, \quad \tau_{x}(f)(y)=\mu_{x} * \mu_{y}\left(P^{t} V_{k}(f)\right), \quad f \in \mathcal{S}\left(\mathbb{R}^{d}\right),
$$

where $*$ is the classical convolution product of measures on $\mathbb{R}^{d}$.

ii) When the multiplicity function satisfies $k(\alpha) \in \mathbb{N} \backslash\{0\}$ for all $\alpha \in R_{+}$, we have

$$
\forall y \in \mathbb{R}^{d}, \quad \tau_{x}(f)(y)=\mu_{x} * \mu_{y}\left({ }^{t} V_{k}(Q(f))\right), \quad f \in \mathcal{S}\left(\mathbb{R}^{d}\right) .
$$

Proof. i) From the relations (4.1) and (1.3), for $f$ in $\mathcal{S}\left(\mathbb{R}^{d}\right)$ we have

$$
\forall x, y \in \mathbb{R}^{d}, \quad \tau_{x}(f)(y)=\int_{\mathbb{R}^{d}} \int_{\mathbb{R}^{d}} V_{k}^{-1}(f)(\xi+\eta) d \mu_{x}(\xi) d \mu_{y}(\eta) .
$$

By using the definition of the classical convolution product of two measures with compact support on $\mathbb{R}^{d}$, we obtain

$$
\forall x, y \in \mathbb{R}^{d}, \quad \tau_{x}(f)(y)=\mu_{x} * \mu_{y}\left(V_{k}^{-1}(f)\right) .
$$

Thus Theorem 5.2 implies the relation (7.1).

ii) The same proof as for i) and Theorem 6.1 give the relation (7.2).

\section{Acknowledgements}

The author would like to thank the referees for their interesting and useful remarks.

\section{References}

[1] Chazarain J., Piriou A., Introduction to the theory of linear partial differential equations, North-Holland Publishing Co., Amsterdam - New York, 1982.

[2] van Diejen J.F., Confluent hypergeometric orthogonal polynomials related to the rational quantum Calogero system with harmonic confinement, Comm. Math. Phys. 188 (1997), 467-497, q-alg/9609032.

[3] Dunkl, C.F., Differential-difference operators associated to reflection groups, Trans. Amer. Math. Soc. 311 (1989), 167-183.

[4] Dunkl C.F., Integral kernels with reflection group invariance, Canad. J. Math. 43 (1991), 1213-1227.

[5] Dunkl C.F., Hankel transform associated to finite reflection groups, Contemp. Math. 138 (1992), $123-138$.

[6] Heckman G.J., An elementary approach to the hypergeometric shift operators of Opdam, Invent. Math. 103 (1991), 341-350.

[7] Humphreys J.E., Reflection groups and Coxeter groups, Cambridge University Press, Cambridge, 1990.

[8] Hikami K., Dunkl operators formalism for quantum many-body problems associated with classical root systems, J. Phys. Soc. Japan 65 (1996), 394-401.

[9] de Jeu M.F.E., The Dunkl transform, Invent. Math. 113 (1993), 147-162.

[10] de Jeu M.F.E., Paley-Wiener theorems for the Dunkl transform, Trans. Amer. Math. Soc. 258 (2006), 4225-4250, math.CA/0404439.

[11] Kakei S., Common algebraic structure for the Calogero-Sutherland models, J. Phys. A: Math. Gen. 29 (1996), L619-L624, solv-int/9608009. 
[12] Lapointe M., Vinet L., Exact operator solution of the Calogero-Sutherland model, Comm. Math. Phys. 178 (1996), 425-452, q-alg/9509003.

[13] Rösler M., Voit M., Markov processes related with Dunkl operators, Adv. in Appl. Math. 21 (1998), 575-643.

[14] Rösler M., Positivity of Dunkl's intertwining operator, Duke. Math. J. 98 (1999), 445-463, q-alg/9710029.

[15] Trimèche K., The Dunkl intertwining operator on spaces of functions and distributions and integral representation of its dual, Integral Transform. Spec. Funct. 12 (2001), 349-374.

[16] Trimèche K., Generalized harmonic analysis and wavelet packets, Gordon and Breach Science Publishers, Amsterdam, 2001.

[17] Trimèche K., Paley-Wiener theorems for the Dunkl transform and Dunkl translation operators, Integral Transform. Spec. Funct. 13 (2002), 17-38. 